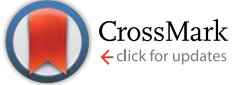

Cite this: J. Mater. Chem. A, 2015, 3 , 8316

\title{
Methane hydrate formation with surfactants fixed on the surface of polystyrene nanospheres
}

\author{
Fei Wang, ${ }^{\text {ab }}$ Sheng-Jun Luo, ${ }^{\text {*a }}$ Shan-Fei Fu, ${ }^{\text {ab }}$ Zhen-Zhen Jia, ${ }^{c}$ Meng Dai, ${ }^{a}$ \\ Chuan-Shui Wang ${ }^{a}$ and Rong-Bo Guo*a
}

\begin{abstract}
To improve the application of surfactants in methane hydrate formation, sodium dodecyl sulfate (SDS) was fixed on the surface of polystyrene nanospheres (named as SDS@PSNS). SDS@PSNS resulted in a shorter induction period of hydrate formation compared to SDS. With SDS@PSNS as a promoter, hydrates formed mainly at the bottom of the reactor with a much higher apparent density and higher methane consumption, and during the hydrate dissociation period, less foam was generated. In addition, the recycling experiments showed high stability and good recycling performance of SDS@PSNS in seven methane hydrate formation-dissociation cycles.
\end{abstract}

Received 10th February 2015

Accepted 6th March 2015

DOI: 10.1039/c5ta01101a

www.rsc.org/MaterialsA

grow upwards on the reactor wall under the capillary effect and

\section{Introduction}

Natural gas hydrates are nonstoichiometric and clathrate crystalline compounds formed by water and natural gas at a suitable temperature and pressure, through gas molecules occupying the cavities formed by water molecules as a result of hydrogenbonding. ${ }^{1}$ Gas hydrates have great potential in natural gas storage and transportation due to the benefits, such as their theoretical storage capacity of up to 180 volumes of natural gas per volume of hydrates and their relatively moderate storage conditions. ${ }^{2}$ However, the industrial application of hydrates in natural gas storage and transportation has been impeded by some problems: the long induction time and slow hydrate growth rate during the hydrate formation process; the separating and packing process of hydrate particles for storage and transportation. ${ }^{3}$

Surfactants, such as SDS have been confirmed to promote hydrate formation efficiently. ${ }^{\mathbf{4 - 1 1}}$ In the deionized water system, according to Englezos et al. ${ }^{12}$ hydrates initially formed as a thin film at the gas/liquid interface and then grew downward as dendrites into the bulk water. The existence of the hydrate film hindered the diffusion of gas into the aqueous phase and therefore resulted in slow hydrate formation. While with SDS as a promoter, hydrates did not form at the gas/liquid interface as dendritic crystals, but on the reactor wall and then grew upwards along the reactor wall and radially along the gas/liquid interface..$^{13}$ Mori et $a l .{ }^{4,5}$ also reported that the hydrates could

${ }^{a}$ Shandong Industrial Engineering Laboratory of Biogas Production and Utilization, Key Laboratory of Biofuels, Qingdao Institute of Bioenergy and Bioprocess Technology, Chinese Academy of Sciences, Qingdao 266101, Shandong, China. E-mail: guorb@qibebt.ac.cn; luosj@qibebt.ac.cn; Fax: +86-532-80662708; +86-53280662750; Tel: +86-532-80662708; +86-532-80662750

${ }^{b}$ University of Chinese Academy of Science, Beijing 10049, China

${ }^{c}$ Guizhou University, Guiyang 550025, China finally accumulated as a highly porous hydrate layer in the upper part of the reactor, which resulted in rapid hydrate formation. However, this might also lead to the hydrates existing in the reactor with a low apparent density. As a result, the space utilization of the reactor might be low and further separation and compaction of the hydrates was inevitable for storage or transportation.

In addition, the promotion effect of surfactants on hydrate formation was influenced by the surfactant concentration significantly and a certain concentration was necessary to obtain an efficient promotion effect. ${ }^{3,6,14}$ Zhong and Rogers ${ }^{3}$ reported that SDS over $242 \mathrm{ppm}$ in the solution produced the efficient promotion of ethane and natural gas hydrate formation. Ganji et al. ${ }^{6}$ found that for cationic and non-ionic surfactants, much higher concentrations (1000 ppm) were needed to produce efficient promotion. However, the solubilities of many surfactants were very low at the hydrate formation conditions, which might lead to precipitation of the surfactants before a high concentration is reached..$^{15}$ Therefore, the application of the surfactants with low solubility in hydrate formation might be hindered. Furthermore, during the hydrate dissociation, a lot of foam would be generated due to the presence of surfactants, which not only affects the application of natural gas hydrates but leads to the outflow of the surfactants.

Recently, fixed bed columns, such as those with a silica sand bed have been employed to promote gas hydrate formation. ${ }^{16-20}$ As the silica sand was usually micro porous, the water could occupy the interstitial spaces of the particles in the bed voids and hydrates could form with the silica sand as the medium. In addition, glass beads ${ }^{21}$ and active carbon ${ }^{22}$ were also used to provide media for hydrate formation. $\mathrm{Li}$ et al. ${ }^{23}$ used surfactant-coated glass beads in clathrate hydrate formation and found that the foreign particles, if properly 
hydrophobized and located, could promote the hydrate nucleation. They proposed that the glass beads could provide nucleation sites for hydrate formation and ordered water molecules and nanobubbles were increased at the hydrophobic surface of the surfactant-coated glass beads, which resulted in fast hydrate nucleation.

In our previous studies, surfactants could be physically or chemically fixed on the surface of polystyrene nano-particles. $^{24,25}$ Surfactants were no longer dispersed in the aqueous phase but arranged on the surface of the particles, which could improve both the solubility and the microscopic concentration of the surfactants. As the nano particles were dispersed in the aqueous phase evenly, a large solid/liquid interface was provided in the aqueous phase. In the present work, SDS was fixed on the surface of polystyrene nanospheres (named as SDS@PSNS) to study the effect of the fixation of SDS on its application in methane hydrate formation.

\section{Experimental}

\section{Materials}

Styrene (A.R.) was purchased from Tianjin Guangcheng Chemical Reagent Co., Ltd (Tianjin, China). Sodium dodecyl sulfate (SDS, A.R.) was provided by Sinopharm Chemical Reagent Co., Ltd (Shanghai, China) and ammonium persulfate (APS, A.R.) was purchased from Hengxing Chemical Reagent Co., Ltd (Tianjin, China). Pyrene ( $\geq 99 \%)$ was purchased from Sigma. Nitrogen and methane with purities of $99.99 \%$ were purchased from Heli Gas Co., Ltd (Qingdao, China).

\section{Preparation of SDS@PSNS}

Styrene was firstly washed three times with a $10 \mathrm{wt} \%$ sodium hydroxide aqueous solution to remove the polymerization inhibitor (hydroquinone), then washed three times with deionized water and stored at $255.15 \mathrm{~K}$ for further use. ${ }^{24}$

SDS@PSNS was prepared by emulsion polymerization with styrene as the monomer, SDS as the emulsifier and APS as the initiator. ${ }^{24}$ The stirring rate was set at $250 \mathrm{rpm}$ and the reaction temperature was maintained at $343.15 \mathrm{~K}$. The reaction time was about $3 \mathrm{~h}$ and the whole polymerization process was nitrogen protected. The recipe and the product names are shown in Table 1 and the schematic diagram of the formation of SDS@PSNS is shown in Scheme 1. During methane hydrate formation, the SDS@PSNS emulsion was diluted with deionized water according to the required SDS concentration.

Table 1 The recipe for the emulsion polymerization and the product names

\begin{tabular}{lllcll} 
Reaction & Product name & SDS $/ g$ & Styrene $/ g$ & APS $/ g$ & Deionized water $/ g$ \\
\hline 1 & SDS@PSNS-1 & 1 & 5 & 0.2 & 95 \\
2 & SDS@PSNS-2 & 1 & 10 & 0.2 & 90 \\
3 & SDS@PSNS-3 & 1 & 20 & 0.2 & 80
\end{tabular}

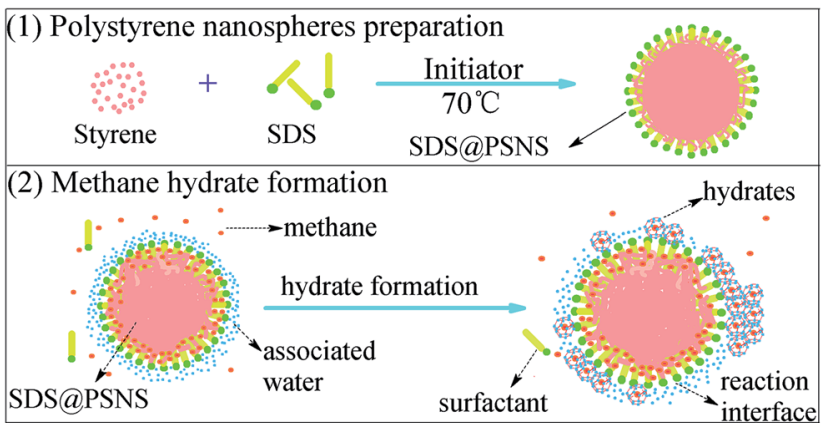

Scheme 1 The schematic diagrams of the formation of SDS@PSNS (1) and the methane hydrate formation with SDSAPSNS as promoter (2).

\section{Characterization of SDS@PSNS}

The morphology of SDS@PSNS was measured via Transmission Electron Microscopy (TEM) using a JEM-1200EX Transmission Electron Microscope made by Japan Electronics Co., Ltd.

The average particle size and particle size distribution index (PDI) of SDS@PSNS were measured using a Malvern Nano-s90 Laser Particle Size Analyzer made by Malvern, UK.

The fixation ratio of SDS on the surface of polystyrene nanospheres was measured by a method using surface tension. The standard curve of SDS concentration $v s$. surface tension was measured firstly, which was linear at low SDS concentration. Then the surface tension of the SDS@PSNS solution was determined and the concentration of free SDS and the fixation ratio were obtained.

The particle number of SDS@PSNS $\left(N_{\mathrm{p}}\right)$ in $1 \mathrm{~mL}$ diluted solution was calculated as follows:

$$
N_{\mathrm{p}}=\frac{3 m \eta}{4 \pi \rho r^{3}}
$$

where $m$ is the weight of the SDS@PSNS solution (g), $\eta$ is the solid content of the SDS@PSNS solution (\%, determined gravimetrically), $\rho$ is the density of SDS@PSNS in solution ( $\mathrm{g}$ $\mathrm{cm}^{-3}$, measured using density bottles) and $r$ is the radius of SDS@PSNS (cm).

The theoretical SDS density at the surface of SDS@PSNS $(\sigma)$ was calculated as follows:

$$
\sigma=\frac{n f \rho r}{3 m \eta}
$$

where $n$ is the overall SDS molar number in the diluted SDS@PSNS solution, $f$ is the fixation ratio of SDS (\%), $\rho$ is the density of SDS@PSNS $\left(\mathrm{g} \mathrm{cm}^{-3}\right), r$ is the radius $(\mathrm{cm}), m$ is the weight ( $\mathrm{g}$ ) and $\eta$ is the solid content of the solution (\%).

\section{Pyrene fluorescence spectra}

The steady-state fluorescence spectra of the reaction solutions were obtained using a Hitachi-4600 Fluorescence Spectrophotometer with pyrene as a probe and Ar light at a wavelength of $337 \mathrm{~nm}$ as the excitation light source at room temperature. ${ }^{26}$ The emission spectra were recorded from $350 \mathrm{~nm}$ to $550 \mathrm{~nm}$ and 
the pyrene concentration in the reaction solutions was $10^{-6} \mathrm{~mol} \mathrm{~L}^{-1}$.

\section{Raman spectroscopy}

The Raman spectra of the reaction solutions were obtained using an in-Via Raman Spectrometer made by Renishaw. The light source was $\mathrm{Ar}^{+}$laser at a wavelength of $532 \mathrm{~nm}$ and a power of $100 \mathrm{~mW}$ and a $10 \times$ objective lens was used. The grating scribed line number was $2200 \mathrm{~mm}^{-1}$ and the slit width was 100 $\mu \mathrm{m}$. The spectral acquisition time was $5 \mathrm{~s}$ and the spectral acquisition range was $2000-3500 \mathrm{~cm}^{-1}$.

\section{Contact angle of the reaction solution on the reactor wall}

To reveal the effect of the fixation of surfactants on the surface tension of the reaction solution the contact angle of the reaction solution on the reactor wall was measured. A droplet of the reaction solution with a volume of $1 \mu \mathrm{L}$ was dropped on the surface of stainless steel, the same material as the reactor and then photos were taken to observe the morphology of the droplet on the surface of the stainless steel.

\section{Methane hydrate formation and dissociation}

Methane hydrate formation was carried out in a $350 \mathrm{~mL}$ stainless steel reactor with a wall jacket connected to the cool bath, as shown in Fig. 1. The reactor was vacuumed and cleaned with methane three times and then $50 \mathrm{~mL}$ of the reaction solution was charged and cooling started. After the reaction temperature $(275.15 \mathrm{~K})$ was reached, the reactor was pressurized with methane to the reaction pressure (6 $\mathrm{MPa}$ ) and the stirrer was set at $200 \mathrm{rpm}$. The temperature and pressure of the reaction system were recorded by the computer during the whole process. After the completion of hydrate formation, the reactor was depressurized and opened immediately and photos were taken of the formed hydrates to observe the morphologies of the hydrates in the reactor. Then the hydrates were taken out and placed on the test bench at room temperature and photos were taken of the hydrates during the dissociation process to observe the morphology changes of the hydrates.

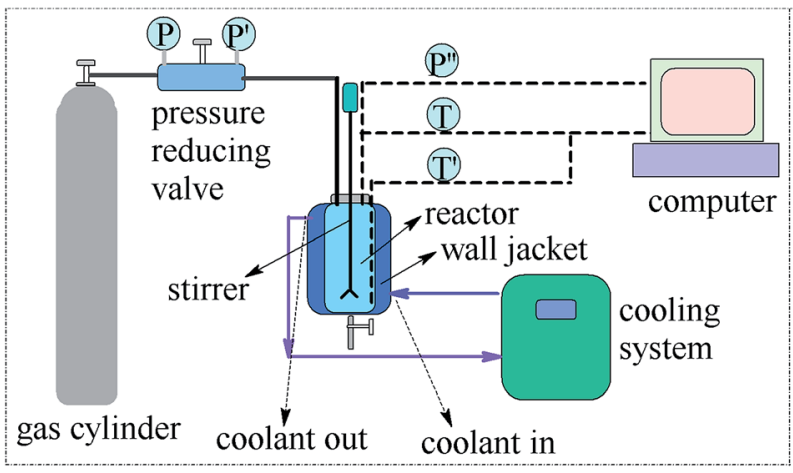

Fig. 1 Schematic diagram of the hydrate formation apparatus.

\section{Calculation of methane consumption}

During methane hydrate formation, the reaction can be expressed as follows:

$$
\mathrm{CH}_{4}+m \mathrm{H}_{2} \mathrm{O} \leftrightarrow \mathrm{CH}_{4} \cdot m \mathrm{H}_{2} \mathrm{O}
$$

where $m$ is the hydration number, which is 5.75 for methane hydrates. ${ }^{27}$

The molar numbers of methane in the reactor at time 0 and $t$ are defined as $n_{0}$ and $n_{t}$ respectively, then the methane consumption $(n)$ at time $t$ can be calculated as follows:

$$
n=n_{0}-n_{t}=\frac{P_{0} V_{0}}{z_{0} R T_{0}}-\frac{P_{t} V_{t}}{z_{t} R T_{t}}
$$

where $P_{0}$ and $P_{t}$ are the pressure of the reactor at time 0 and $t ; V_{0}$ and $V_{t}$ are the volume of the gas phase in the reactor at time 0 and $t ; R$ is the universal gas constant; $T_{0}$ and $T_{t}$ are the temperatures in the reactor at time 0 and $t ; z_{0}$ and $z_{t}$ are the compressibility factors at time 0 and $t$, which are calculated using the Pitzer correlations for the compressibility factor ${ }^{28}$ and the derived calculation formula is shown as follows:

$$
\begin{aligned}
z_{t}= & 1+\left[0.083-0.422 \times\left(\frac{T_{\mathrm{c}}}{T_{t}}\right)^{1.6}\right] \frac{P_{t} T_{\mathrm{c}}}{P_{\mathrm{c}} T_{t}} \\
& +\omega\left[0.139-0.172 \times\left(\frac{T_{\mathrm{c}}}{T_{t}}\right)^{4.2}\right] \frac{P_{t} T_{\mathrm{c}}}{P_{\mathrm{c}} T_{t}}
\end{aligned}
$$

where $T_{\mathrm{c}}$ is $190.6 \mathrm{~K}, P_{\mathrm{c}}$ is $4.599 \mathrm{MPa}$ and $\omega$ is 0.012 for methane.

It should be noted that with the formation of hydrates, $V_{t}$ changed due to the different densities of water and hydrates. $V_{t}$ can be calculated as follows:

$$
V_{t}=V_{0}-\left(V_{\mathrm{h}}-V_{\mathrm{cw}}\right)=V_{0}-m \times n \times \Delta V
$$

where $V_{\mathrm{h}}$ is the volume of hydrates in the reactor at time $t ; V_{\mathrm{cw}}$ is the volume of water consumed at time $t ; \Delta V$ is the molar volume difference between methane hydrates and water, which has been reported as $4.6 \mathrm{~cm}^{3}$ per mole of water; ${ }^{29} n$ is the amount of methane consumed at time $t$ and $m$ is hydration number.

Then from eqn (3)-(6) $n$ can be calculated as follows:

$$
n=\frac{\frac{P_{0} V_{0}}{z_{0} R T_{0}}-\frac{P_{t} V_{0}}{z_{t} R T_{t}}}{1-\frac{P_{t} \Delta V m}{z_{t} R T_{t}}}
$$

\section{Recycling of SDS@PSNS in methane hydrate formation}

Cyclic formation of methane hydrates with SDS@PSNS-2 as a promoter was performed at $275.15 \mathrm{~K}$ and an initial pressure of 6 $\mathrm{MPa}$. After the hydrate formation was accomplished, the reactor was heated to $293.15 \mathrm{~K}$ and kept at this temperature for $2 \mathrm{~h}$ to make the hydrates thoroughly dissociated and to avoid the memory effect. Then cooling was started and a new round of hydrate formation began. After several hydrate formationdissociation cycles, the SDS@PSNS-2 solution was sampled and characterized. 


\section{Results and discussion}

\section{Characterization of SDS@PSNS}

Fig. 2 shows the morphologies of the SDS@PSNS particles, all the SDS@PSNS particles appeared as uniform spheres at the nanoscale. Fig. 3 shows the particle size and PDI of SDS@PSNS in aqueous solution, which denoted the high monodispersity of SDS@PSNS. Table 2 shows some colloidal features of different SDS@PSNS solutions at the same SDS concentration $\left(1 \mathrm{mmol} \mathrm{L}^{-1}\right)$. More styrene used in the polymerization resulted in a higher surfactant fixation ratio and total surface area of SDS@PSNS, but a lower particle number and surfactant density on the surface of SDS@PSNS.

\section{Methane hydrate formation}

The induction time was defined as the time from charging methane into the reactor until obvious pressure decrease or gas consumption was observed. ${ }^{30}$ Fig. 4 shows the evolution of the methane consumption during hydrate formation with different reaction solutions. During hydrate formation with SDS, an obvious induction period was observed and rapid hydrate growth was achieved after the induction period. However, for hydrate formation with SDS@PSNS-2 and SDS@PSNS-3, continuous methane consumption was observed from the beginning of the recorded time. Hydrates formed very slowly during the initial $400 \mathrm{~min}$ and $600 \mathrm{~min}$ methane hydrate formation with SDS@PSNS-2 and SDS@PSNS-3 respectively. Then rapid hydrate growth was observed. In addition, it should be noted that even with relatively lower hydrate growth rate, all the SDS@PSNSs resulted in higher methane consumption compared with SDS.

According to Hashemi et al., ${ }^{31}$ the molar number of solubilized methane in a $50 \mathrm{~mL}$ solution in the presence of hydrates was about 0.005 . Taking the maximum error of the recorded pressure into consideration (the molar error was calculated as 0.005), the methane consumption over $0.01 \mathrm{~mol}$ could be considered as the completion of the induction period and the beginning of hydrate growth in this work.

Table 3 shows the induction time of methane hydrate formation with different accelerants. Given the stochasticity of
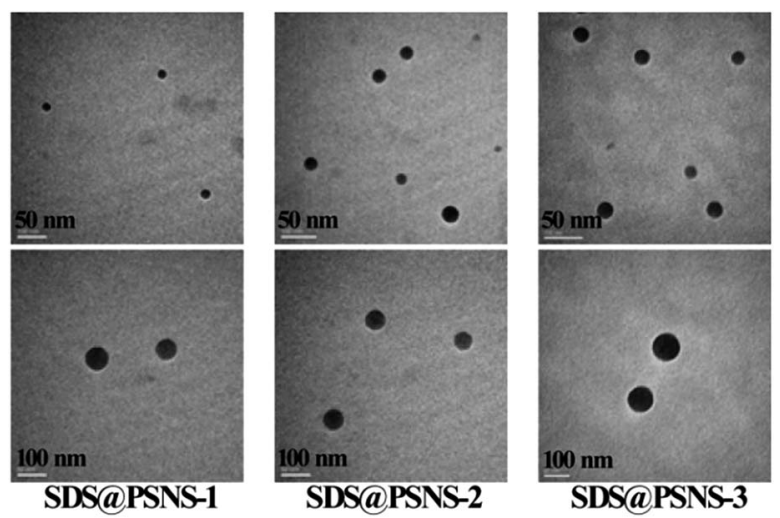

Fig. 2 TEM photos of SDS@PSNS

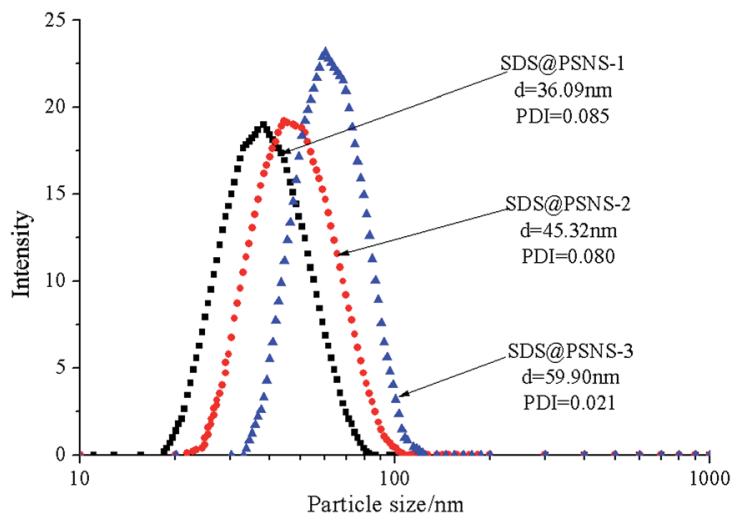

Fig. 3 Particle size $(d)$ and PDI of SDS@PSNS in aqueous solution.

hydrate formation, each experiment was carried out three times. For comparison, hydrate formation with deionized water was carried and no obvious pressure decrease was observed within 3 days. In addition, polystyrene nanosphere powder (PSNS powder) with the same weight as SDS@PSNS-3 was also applied in methane hydrate formation to exclude the effects of the pure polystyrene nanospheres, which showed no promotion of methane hydrate formation.

As shown in Table 3, the use of SDS@PSNS resulted in a much shorter induction period than SDS. During hydrate formation with SDS, SDS molecules were dispersed in the aqueous phase. Therefore, SDS concentration showed a great influence on the promotion effect. When SDS concentration was low $\left(0.5 \mathrm{mmol} \mathrm{L}^{-1}\right)$, there were not enough SDS molecules to show efficient promotion of methane hydrate formation. However, in the SDS@PSNS solution, SDS molecules were arranged on the surface of the polystyrene nanospheres, which could improve the microscopic concentration of SDS on the surface of the nanospheres. As a result, SDS@PSNS could lead to a much shorter induction period than SDS, especially at low concentration.

In addition, different SDS@PSNSs also showed unequal promotion effects (Fig. 4). During the initial $300 \mathrm{~min}$ of hydrate formation, the use of SDS@PSNS-3 resulted in the highest formation rate, while during the rapid hydrate growth period, using SDS@PSNS-1 led to the highest formation rate. As shown in Table 2, SDS@PSNS-3 provided the largest surface area in the

Table 2 Colloidal features of different SDS@PSNS solutions at the same surfactant concentration $\left(c_{\mathrm{s}}\right)^{a}$

\begin{tabular}{llllll}
\hline & & $\begin{array}{l}N_{\mathrm{p}} \\
\left(\times 10^{13}\right)\end{array}$ & $f(\%)$ & $s\left(\mathrm{~m}^{2}\right)$ & $\begin{array}{l}\sigma \\
\left(10^{15} \mathrm{~cm}^{-2}\right)\end{array}$ \\
\hline SDS@PSNS-1 & 1 & 6.67 & 81.96 & 0.27 & 3.62 \\
SDS@PSNS-2 & 1 & 6.08 & 88.15 & 0.39 & 2.70 \\
SDS@PSNS-3 & 1 & 4.99 & 93.78 & 0.56 & 2.01
\end{tabular}

${ }^{a} N_{\mathrm{p}}$ - particle number in $1 \mathrm{~mL}$ diluted SDS@PSNS solution, $f$ surfactant fixation ratio, $\sigma$ - surfactant density at the surface of SDS@PSNS, $s$ - total surface area of SDS@PSNS in $1 \mathrm{~mL}$ of diluted SDS@PSNS solution. 


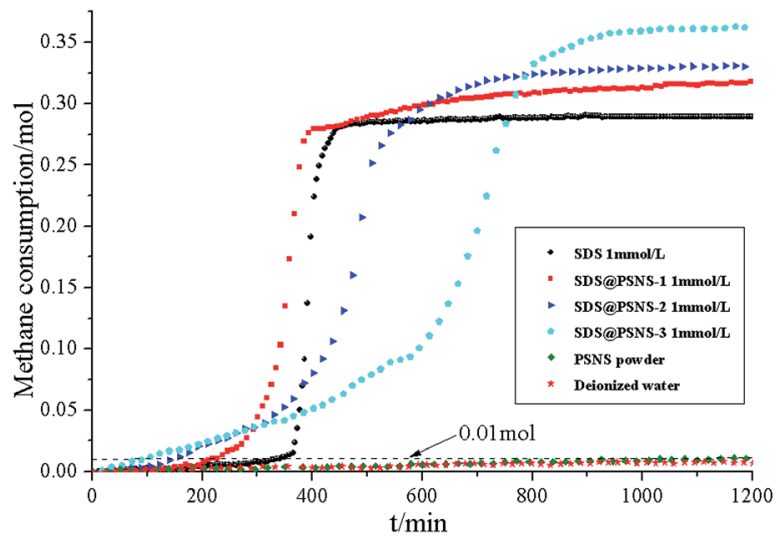

Fig. 4 The evolution of the methane consumption during hydrate formation with different reaction solutions (the initial pressure was 6 $\mathrm{MPa}$ and the cold bath temperature was $275.15 \mathrm{~K}$ ).

Table 3 The induction time of methane hydrate formation with different accelerants

\begin{tabular}{lllll}
\hline & & \multicolumn{3}{c}{ Induction time/min } \\
\cline { 3 - 5 } Accelerant & $c_{\mathrm{s}}{ }^{a}\left(\mathrm{mmol} \mathrm{L}^{-1}\right)$ & 1 & 2 & 3 \\
\hline SDS & 0.5 & $-{ }^{b}$ & - & - \\
& 1 & 350 & - & 486 \\
& 2 & 185 & 570 & 409 \\
SDS@PSNS-1 & 0.5 & 209 & 162 & 115 \\
& 1 & 216 & 55 & 76 \\
SDS@PSNS-2 & 2 & 85 & 80 & 84 \\
SDS@PSNS-3 & 1 & 140 & 65 & 63 \\
PSNS powder & 1 & 87 & 128 & 90 \\
Deionized water & & - & - & - \\
& & - & - & -
\end{tabular}

${ }^{a}$ The concentration of SDS@PSNS was determined as the overall SDS concentration in the SDS@PSNS solution. ${ }^{\circ}$ "-” indicates that no obvious pressure decrease was detected within 1200 minutes.

reaction solution, which might result in the most hydrate nucleation sites. Therefore, during the initial $300 \mathrm{~min}$, using SDS@PSNS-3 resulted in the highest hydrate formation rate. Table 2 also shows that SDS@PSNS-1 produced the highest particle concentration and SDS density at the nanosphere surface. As a result, much more hydrate particles could be formed during the rapid hydrate growth period in the SDS@PSNS-1 solution, which might result in the highest hydrate formation rate.

\section{Methane hydrate growth pattern and dissociation morphology}

Fig. 5 (middle column) shows the morphologies of the methane hydrates in the reactor after the reactor was opened. The reactor was depressurized quickly after the completion of hydrate formation and the temperature in the reactor decreased rapidly until lower than $273.15 \mathrm{~K}$. Therefore, the melting of the hydrates could be neglected and the morphology of the hydrates
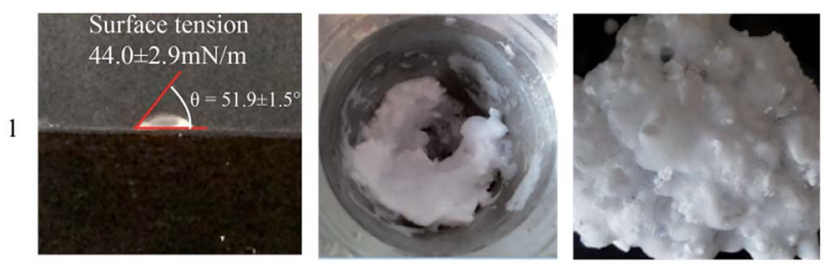

2
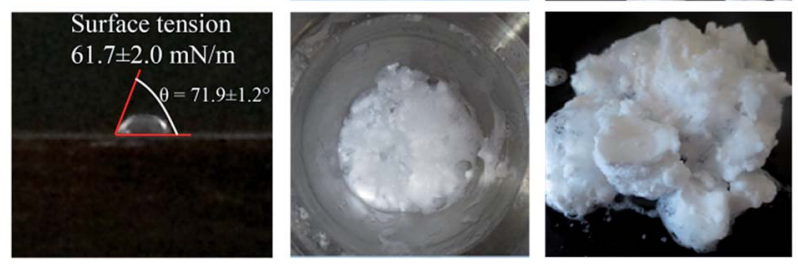

3
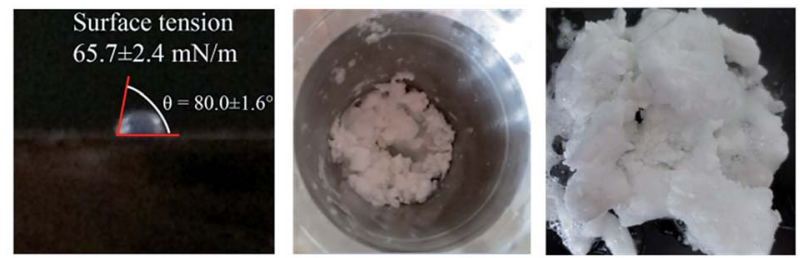

4
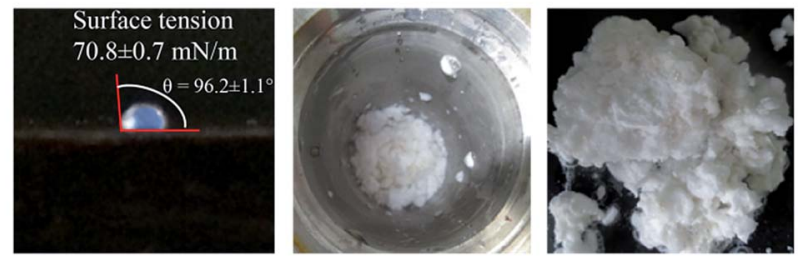

Fig. 5 Left column: contact angles $(\theta)$ of SDS and SDS@PSNS solutions $\left(1 \mathrm{mmol} \mathrm{L}{ }^{-1}\right)$ on the reactor wall; middle column: methane hydrate morphologies in the reactor after the completion of hydrate formation; right column: hydrate morphologies during the dissociation period (photos were taken $3 \mathrm{~min}$ after being taken out); 1-SDS, 2SDS@PSNS-1, 3-SDS@PSNS-2, 4-SDS@PSNS-3; the surface tension of deionized water was determined to be $77.6 \pm 1.6 \mathrm{mN} \mathrm{m}^{-1}$ and the contact angle of deionized water was determined to be $100.4 \pm 1.2^{\circ}$.

observed after the reactor was opened could denote the morphology of hydrates in the reactor after hydrate formation. During hydrate formation with SDS as a promoter, hydrates mainly formed on the reactor wall and grew upwards to the upper edge of the reactor, which was similar to previous reports. ${ }^{4,5}$ While when SDS@PSNS was used, hydrates mainly formed at the bottom of the reactor.

During hydrate growth with SDS@PSNS, as the surfactants were fixed, a higher surface tension of the SDS@PSNS solution was obtained compared with the SDS solution, which could also be revealed by the contact angle of the reaction solution on the reactor wall, ${ }^{32}$ as shown in Fig. 5 . The high surface tension and contact angle denoted poor wettability of the SDS@PSNS solution on the reactor wall, ${ }^{33}$ which might not be conducive to the upward growth of the hydrates on the reactor wall. Furthermore, SDS@PSNS was dispersed in the solution uniformly during hydrate formation and hydrates might form with the nanospheres as media. Therefore, hydrates mainly formed in the bulk of the SDS@PSNS solution and the continuous growth and agglomeration of the formed methane hydrates finally resulted in the completion of methane hydrate formation at the bottom of the reactor. A higher surfactant fixation ratio was 
more conducive to hydrate formation at the bottom of the reactor due to the higher surfactant fixation ratio and larger contact angle. In addition, it should be noted that the hydrates formed with SDS@PSNS as a promoter exhibited much higher apparent density in comparison to the hydrates formed with SDS as a promoter, as shown in Fig. 5.

Fig. 5 (right column) also shows that a lot of foam was generated from the hydrates formed with SDS, while minimal foam was generated when surfactants were fixed on the surface of polystyrene nanospheres. During the dissociation of hydrates with SDS, the hydrates melted into the water and the surfactants dissolved in the water again. Afterwards, with the continuous release of methane a lot of foam was generated. While when the surfactants were fixed on the surface of SDS@PSNS, few free surfactants existed in the water melted from the hydrates. Therefore, minimal foam was generated.

\section{Promotion mechanism of SDS@PSNS on methane hydrate formation}

For hydrate formation with SDS as an accelerant, surfactants could promote hydrate formation by adsorbing onto the hydrate nuclei, which might result in a decrease in the hydrate nuclei/ liquid interfacial energy. ${ }^{34}$ However, when the surfactants are fixed on the surface of polystyrene nanospheres, the promotion mechanism might be different.

On one hand, as previously reported the hydrate formers could dissolve in the hydrophobic domains formed by the surfactants adsorbed on the hydrate surface, ${ }^{34,35}$ the methane molecules could dissolve in the alkyl chains of the surfactants and the macromolecular chains of the polymers on the SDS@PSNS surface (Scheme 1). This view was supported by the pyrene fluorescence measurement, which has been successfully applied to detect the hydrophobic domains on the particle surface. ${ }^{32}$ The ratio of the intensities of the third peak $\left(I_{3}, \lambda=384 \mathrm{~nm}\right)$ and the first peak $\left(I_{1}, \lambda=374 \mathrm{~nm}\right)$ could denote the polarities of the microdomains, which vary from 0.50 to 0.80 in polar solvents and 1.65 to 1.75 in hydrocarbon solvents. As shown in Fig. 6A, the value of $I_{3} / I_{1}$ in the SDS solution is 0.7 , which is almost the same as that in deionized water (0.69), indicating that no hydrophobic domains were formed in the SDS solution. However, the value of $I_{3} / I_{1}$ in the SDS@PSNS-3 solution is 1.09 , denoting the existence of hydrophobic domains with high nonpolarity in the solution, which could be provided by the surfactants alkyl chains and the polymer macromolecular chains on the SDS@PSNS-3 surface.

On the other hand, as discussed by Chrystal et al. ${ }^{36}$ water molecules could be associated at the surface of SDS micelles through the hydrogen bonds between the oxygen atoms of the sulfate groups and the hydrogen atoms of the water molecules. Therefore, a layer of water molecules could be associated on the surface of the SDS@PSNS in the solution as the structure of the SDS@PSNS was similar to that of the micelles. Fig. 6B shows that the intensities in the Raman spectrum of the SDS@PSNS-3 solution were higher than those of the SDS solution and deionized water at Raman shifts of 3241 and $3415 \mathrm{~cm}^{-1}$, which were the symmetric and asymmetric stretching vibration peaks
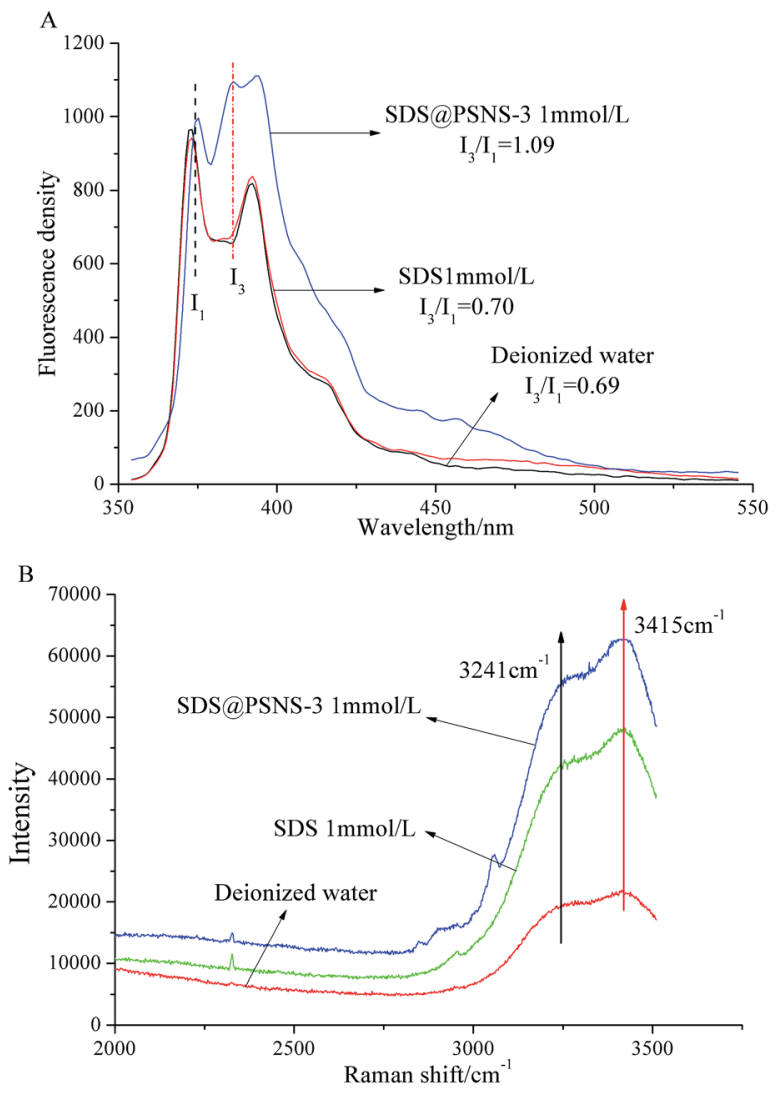

Fig. 6 The pyrene fluorescence emission spectra (A) and Raman spectra (B) of deionized water, SDS solution $\left(1 \mathrm{mmol} \mathrm{L}^{-1}\right)$ and SDS@PSNS-3 solution $\left(1 \mathrm{mmol} \mathrm{L}^{-1}\right)$.

of $\mathrm{O}-\mathrm{H}$ with hydrogen bonding. The higher intensities of the two peaks indicated the stronger hydrogen bonds in the SDS@PSNS-3 solution, which could be caused by the association of water molecules on the surface of SDS@PSNS-3.

As a result, hydrates might firstly form through the reaction between the methane molecules dissolved in the surface of the SDS@PSNS and the water molecules associated by the sulfate groups, resulting in hydrate formation on the SDS@PSNS surface and subsequently the formation of hydrate particles with SDS@NSPS as nuclei. Then the continuous growth and agglomeration of the hydrate particles finally results in the formation of gas hydrates.

To verify the hydrate formation mechanism in the presence of SDS@PSNS, the hydrate formation-dissociation and freezing-thawing processes of the SDS@PSNS-3 solution were conducted for comparison. As shown in Fig. 7A and B, the SDS@PSNS-3 solution became turbid after the freeze-thaw process and stratified after centrifugation. According to the literature, ${ }^{37}$ during the freezing process, ice crystals formed in the aqueous phase, resulting in the continuous increase in the concentration of SDS@PSNS-3 in the unfrozen water. Finally, the SDS@PSNS-3 separated out from the aqueous phase and agglomerated under the extrusion caused by the volume expansion of the water that was undergoing freezing. During the melting process, the SDS@PSNS-3 could not detach from the 


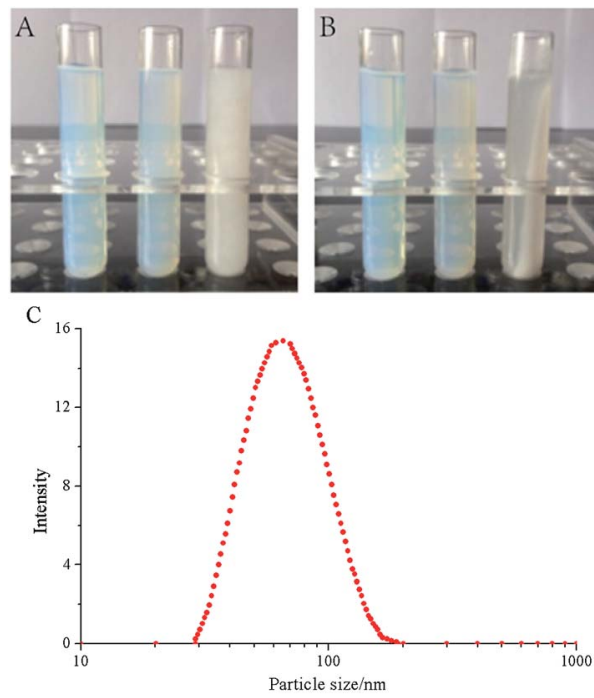

Fig. 7 Photos of SDSAPSNS-3 solutions before centrifugation (A) and after centrifugation at 2000 rpm for 3 minutes (B) (for both $A$ and $B$, the left tube is the original SDSAPSNS-3 solution, the middle one is the SDSQPSNS-3 solution after hydrate formation-dissociation and the right one is the SDSQPSNS-3 solution after freezing-thawing); C: the particle size and PDI of the SDSAPSNS-3 solution after hydrate formation-dissociation $(d=63.99 \mathrm{~nm}, \mathrm{PDI}=0.160)$.

agglomerates, leading to a turbid SDS@PSNS-3 solution which became stratified after centrifugation.

However, the hydrate formation-dissociation process did not impact the SDS@PSNS-3 solution greatly. Fig. 7C shows that the average particle size and PDI of the SDS@PSNS-3 did not change too much, indicating a formation mechanism that was different from freezing. As discussed above, hydrate formation firstly took place on the surface of SDS@PSNS-3, resulting in the formation of hydrate particles with SDS@PSNS-3 as nuclei. Consequently, the SDS@PSNS-3 could not separate out from the aqueous phase and agglomerate together. Then during the dissociation process, the SDS@PSNS-3 could be released from the hydrates and disperse in the aqueous phase as before, which was consistent with the experimental results.

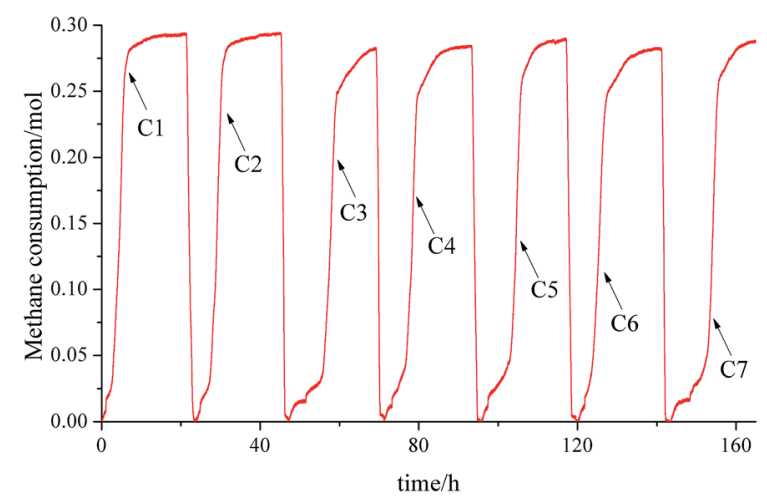

Fig. 8 Cyclic formation-dissociation of methane hydrates with the SDSaPSNS-2 solution $\left(1 \mathrm{mmol} \mathrm{L}^{-1}\right)$ (the ascending curves represent the hydrate formation and declining curves represent the hydrate dissociation).

\section{Recycling of SDS@PSNS in methane hydrate formation}

The stability and recycling performance of SDS@PSNS in methane hydrate formation play a critical role in the industrial application of methane hydrates. Fig. 8 shows the evolution of methane consumption in seven methane hydrate formationdissociation cycles with the SDS@PSNS-2 solution. All the cycles exhibited the rapid formation and high gas consumption of methane hydrates, indicating the good recycling performance of SDS@PSNS in methane hydrate formation.

\section{Conclusions}

SDS was fixed on the surface of polystyrene nanospheres in methane hydrate formation and an efficient promotion effect was observed. With SDS@PSNS as a promoter, hydrates did not formed on the reactor wall, but at the bottom of reactor with a higher apparent density, which could be conducive to the separation of hydrates from the unreacted water and the compaction for storage and transportation. The hydrate formation-dissociation cycles showed the high stability and good recycling performance of SDS@PSNS in methane hydrate formation.

\section{Acknowledgements}

This work was supported by the Taishan Scholar Program of Shandong Province, the National Natural Science Foundation of China (31101918, 21307143), 863 Project (2012AA052103), Key Projects in the National Science and Technology Pillar Program (20140015) and the Qingdao Science and Technology and People's Livelihood Project (14-2-3-69-nsh).

\section{Notes and references}

1 M. R. Walsh, C. A. Koh, E. D. Sloan, A. K. Sum and D. T. Wu, Science, 2009, 326, 1095-1098.

2 W. X. Wang, C. L. Bray, D. J. Adams and A. I. Cooper, J. Am. Chem. Soc., 2008, 130, 11608-11609.

3 Y. Zhong and R. E. Rogers, Chem. Eng. Sci., 2000, 55, 41754187.

4 K. Okutani, Y. Kuwabara and Y. H. Mori, Chem. Eng. Sci., 2008, 63, 183-194.

5 K. Watanabe, S. Imai and Y. H. Mori, Chem. Eng. Sci., 2005, 60, 4846-4857.

6 H. Ganji, M. Manteghian, M. R. Omidkhah and M. H. Rahimi, Fuel, 2007, 86, 434-441.

7 J. S. Zhang, S. Lee and J. W. Lee, Ind. Eng. Chem. Res., 2007, 46, 6353-6359.

8 Y. A. Kwon, J. M. Park, K. E. Jeong, C. U. Kim, T. W. Kim, H. J. Chae, S. Y. Jeong, J. H. Yim, Y. K. Park and J. D. Lee, J. Ind. Eng. Chem., 2011, 17, 120-124.

9 N. Ando, Y. Kuwabara and Y. H. Mori, Chem. Eng. Sci., 2012, 73, 79-85.

10 C. Lo, J. Zhang, P. Somasundaran and J. W. Lee, J. Colloid Interface Sci., 2012, 376, 173-176. 
11 J. S. Zhang, S. Lee and J. W. Lee, J. Colloid Interface Sci., 2007, 315, 313-318.

12 J. D. Lee, M. Song, R. Susilo and P. Englezos, Cryst. Growth Des., 2006, 6, 1428-1439.

13 J. Yoslima, P. Linga and P. Englezos, J. Cryst. Growth, 2010, 313, 68-80.

14 V. Jonathan and S. Phillip, Ind. Eng. Chem. Res., 2012, 51, 13144-13149.

15 P. P. Di, S. Arca, R. Germani and G. Savelli, Chem. Eng. Sci., 2005, 60, 4141-4145.

16 D. L. Zhong, N. Daraboina and P. Englezos, Energy Fuels, 2013, 27, 4581-4588.

17 P. Linga, N. Daraboina, J. A. Ripmeester and P. Englezos, Chem. Eng. Sci., 2012, 68, 617-623.

18 A. Adeyemo, R. Kumar, P. Linga, J. Ripmeester and P. Englezos, Int. J. Greenhouse Gas Control, 2010, 4, 478-485.

19 D. Sun and P. Englezos, Int. J. Greenhouse Gas Control, 2014, 25, 1-8.

20 M. J. Yang, Y. C. Song, L. L. Jiang, X. J. Wang, W. G. Liu, Y. C. Zhao, Y. Liu and S. R. Wang, J. Ind. Eng. Chem., 2014, 20, 322-330.

21 M. J. Yang, Y. C. Song, L. L. Jiang, Y. Liu and X. J. Wang, Sep. Purif. Technol., 2015, 141, 170-178.

22 X. X. Zhang, H. Liu, C. Y. Sun, P. Xiao, B. Liu, L. Y. Yang, C. H. Zhan, X. Q. Wang, N. Li and G. J. Chen, Sep. Purif. Technol., 2014, 130, 132-140.

23 H. J. Li and L. G. Wang, Fuel, 2015, 140, 440-445.

24 F. Wang and K. J. Fang, Colloid Polym. Sci., 2014, 292, 14491455.
25 F. Wang and K. J. Fang, Colloid Polym. Sci., 2014, 292, 23752383.

26 J. S. Zhang, C. Lo, P. Somasundaran, S. Lu, A. Couzis and J. W. Lee, J. Phys. Chem. C, 2008, 112, 12381-12385.

27 E. D. Sloan and C. A. Koh, in Clathrate Hydrates of Natural Gases, ed. H. Heinemann and J. G. Speight, CRC press, Boca Raton, 3rd edn, 2007, ch. 2, pp. 60-63.

28 J. M. Smith, N. H. C. Van and M. M. Abbott, in Introduction to Chemical Engineering Thermodynamics, McGraw-Hill Education, Singapore, 6th edn, 2001, pp. 96-98.

29 Y. F. Makogon, in Hydrates of hydrocarbons, Pennwell Books, Oklahoma, 1997, pp. 35-37.

30 E. D. Sloan and C. A. Koh, in Clathrate Hydrates of Natural Gases, ed. H. Heinemann and J. G. Speight, Boca Raton, CRC press, 2007, pp. 131-133.

31 S. Hashemi, A. Macchi, S. Bergeron and P. Servio, Fluid Phase Equilib., 2006, 246, 131-136.

32 M. Rankl, S. Laib and S. Seeger, Colloids Surf., B, 2003, 30, 177-186.

33 F. M. Fowkes and W. A. Zisman, in Contact angle, wettability, and adhesion, 1964.

34 J. S. Zhang, C. Lo, P. Somasundaran, S. Lu, A. Couzis and J. W. Lee, J. Phys. Chem. C, 2008, 112, 12381-12385.

35 C. Lo, J. S. Zhang, A. Couzis, P. Somasundaran and J. W. Lee, J. Phys. Chem. C, 2010, 114, 13385-13389.

36 D. B. Chrystal, S. Sanjib, L. Max, L. P. Berkowitz and D. E. F. Malcolm, J. Phys. Chem. B, 2002, 106, 10902-10907.

37 Z. Zong, S. Wall, Y. Z. Li, J. Ruiz, H. Adam, C. Badre and F. Trezzi, Prog. Org. Coat., 2010, 72, 115-119. 